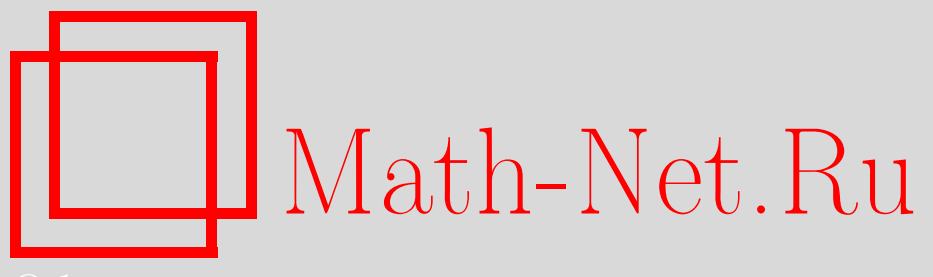

С. В. Жуленев, О больших уклонениях, II, Теория вероятн. и ее примен., 2004, том 49, выпуск 4, 672694

DOI: https://doi.org/10.4213/tvp188

Использование Общероссийского математического портала MathNet.Ru подразумевает, что вы прочитали и согласны с пользовательским соглашением

http://www . mathnet.ru/rus/agreement

Параметры загрузки:

IP : 52.87 .193 .239

26 апреля 2023 г., 13:50:46

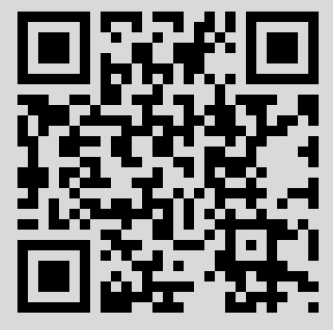


(C) $2004 \mathrm{r}$.

\author{
ЖУЛЕНЕВ С. В.*
}

\title{
О БОЛЬШИХ УКЛОНЕНИЯХ, II
}

Метод Эшера-Крамера позволяет получать представления больших уклонений и без условия Крамера. При этом для нормированных сумм независимых и одинаково распределенных случайных величин при достаточно большом числе слагаемых они оказываются мало отличаюшимися от тех, которые справедливы при выполнении этого жесткого условия.

Ключевые слова и фразы: обобщенное преобразование Крамера, усеченная экспонента, условные математические ожидания.

Введение. Практически с момента возникновения проблематики больших уклонений (БУ), т.е. уже более 60 лет, на повестке дня стоит один вопрос: существует ли представление БУ (ПБУ) без условия Крамера, в определенном смысле аналогичное тому, которое имеет место при этом условии, и если нет, то почему? В настоящей работе, которая является продолжением [1], предпринята попытка разобраться в этой ситуации и показано, что такое представление существует.

Условие, заменяющее условие Крамера, естественно и сводится к существованию некоторого момента у слагаемых нормированной суммы. Однако форму представления, которую можно было бы называть «аналогичной крамеровской», фактически подсказал нетрадиционный взгляд на метод Крамера, предложенный в [1] (причем по ряду причин оказалась удобной форма, используемая в [2]). Так что становится понятным, почему так долго не удавалось ответить на указанный вопрос. Конечно, если автор не ошибается, и то, что ему ничего не известно о каких-то ответах на него, действительно означает, что эти ответы и не были даны.

С математической точки зрения основную часть работы составляет доказательство одной теоремы. К сожалению, оно оказалось весьма громоздким, и даже формулировка потребовала специальной подготовки, проведенной в п. 1. Поэтому его пришлось упрощать, выделяя узловые моменты и вынося их доказательство за рамки непосредственного

* Московский государственный университет им. М.В. Ломоносова, механикоматематический факультет, Ленинские горы, 119992 Москва, Россия; е-таil: zhulen@mech.math.msu.su 
обоснования теоремы, приведенного в п. 2, - они помещень в пп. 3-5. Мотивировать же тот факт, что предложенное представление «аналогично крамеровскому», наоборот, совсем необязательно. Достаточно лишь взглянуть на эти два представления (использованное в [2] приведено в п. 7). Но в п. 7 все же дан и сравнительный анализ этих представлений, поскольку представляет интерес, сближаются ли в определенном смысле рассматриваемые представления при увеличении числа слагаемых нормированной суммы или «сближении» используемого условия с условием Крамера. Наконец, в п. 6 помешены несколько утверждений, полезных при вычислении некоторых элементов нашего представления, поскольку основной целью нетрадиционного подхода является все же не столько асимптотика «хвоста распределения», сколько его запись в виде конкретной функции на конкретном интервале.

\section{1. Одинаковые слагаемые. Каноническое представление.} Рассмотрим получение ПБУ в простейшем случае нормированной суммы $S_{n} / s_{n}$ независимых одинаково распределенных случайных величин (н.о.р.с.в.) $\left(X_{l}\right)_{l \geqslant 1}$; пусть, как и обычно, $S_{n}=\sum_{1}^{n} X_{l}, s_{n}^{2}=\mathrm{D} S_{n}$. При этом по-прежнему будем считать, что все наши (одномерные) с.в. несмешены, как и в ч. I, но теперь имеют лишь конечное число моментов. Точнее говоря, допустим, что

$$
\begin{gathered}
\mathbf{E} X_{1}=0, \quad \mathbf{D} X_{1}=d^{2}>0, \\
\alpha_{2 s+2}<\infty
\end{gathered}
$$

при некотором фиксированном целом $s \geqslant 1$; здесь и ниже $\alpha_{k}=\mathbf{E} X_{1}^{k}$.

Однако прежде чем перейти к получению искомого представления, проведем небольшую подготовку. А именно, уточним вид используемого обобшенного преобразования Крамера (ОПК; см. [1]), введем большую часть обозначений и установим соотношение, которое назовем «каноническим». Дело в том, что его можно считать лежащим в основе любых ПБУ нашего типа. Как тех, которые устанавливаются при условии Крамера, так и тех, которые мы собираемся доказывать с его помощью.

1.1. Используемые аналоги преобразования Крамера. Как и в ч. I, договоримся считать всюду ниже основным параметр $\lambda$, вообще говоря, пробегающий положительную ось. Но сначала предположим, что произвольное $\lambda>0$ фиксировано, и введем последовательность независимых одномерных с.в. $Y_{l}=\zeta\left(\lambda ; X_{l} / s_{n}\right)$, которые, как и в ч. I, будем называть ОПК с.в. $X_{l} / s_{n}$ с функцией $g_{\lambda}(t)=e_{2 s}(\lambda t), e_{s}(t)=1+t+\cdots+t^{s} / s !$. Означать же это будет, что их распределение задается равенством

$$
d \mathbf{P}\left\{Y_{l}<t\right\}=e_{2 s}(\lambda t) \varphi_{2 s}^{-1}(z) d \mathbf{P}\left\{X_{l}<s_{n} t\right\}
$$

здесь и ниже $\varphi_{s}(\lambda)=\mathbf{E} e_{s}\left(\lambda X_{l}\right)=\sum_{0}^{s} \alpha_{k} \lambda^{k} / k !, z=\lambda / s_{n}$. 
Введенные выше н.о.р.с.в. $Y_{l}, 1 \leqslant l \leqslant n$ (а их сушествование вытекает из теоремы Колмогорова), фактически являются основными «кирпичиками» проводимого ниже анализа, поскольку в дальнейшем потребуется лишь преобразование, являюшееся покоординатным произведением выражений (3):

$$
\prod_{l=1}^{n} d \mathbf{P}\left\{Y_{l}<y_{l}\right\}=\varphi_{2 s}^{-n}(z) \prod_{l=1}^{n} e_{2 s}\left(\lambda y_{l}\right) d \mathbf{P}\left\{X_{l}<s_{n} y_{l}\right\} .
$$

Ясно, что определенное таким образом распределение $n$-мерного вектора $Y=\left(Y_{1}, \ldots, Y_{n}\right)$ с независимыми компонентами можно называть OПК $n$-мерного вектора $X / s_{n}=\left(X_{1}, \ldots, X_{n}\right) / s_{n}$ с функцией $G_{n}(\lambda ; y)=$ $\prod_{l=1}^{n} e_{2 s}\left(\lambda y_{l}\right), y=\left(y_{1}, \ldots, y_{n}\right)$. Именно с помощью преобразования (4) мы и будем записывать искомую вероятность $\mathbf{P}\left\{S_{n}>s_{n} x\right\}$, используя несколько новых и старых обозначений:

$$
\begin{gathered}
m_{l}=\mathbf{E} Y_{l} \equiv m, \quad \zeta=\sum_{1}^{n} Y_{l}, \quad x=\mathbf{E} \zeta, \quad \sigma^{2}=\mathbf{D} \zeta, \quad \eta=\prod_{l=1}^{n} e_{2 s}^{-1}\left(\lambda Y_{l}\right), \\
\bar{Y}_{l}=\frac{Y_{l}-m}{\sigma}, \quad H=\sum_{1}^{n} \bar{Y}_{l}, \quad H(t)=\mathbf{P}\{H<t\}=\mathbf{P}\{\zeta<x+\sigma t\}, \\
\nu=\sup _{t}|H(t)-\Phi(t)|, \quad \Phi(t)=\frac{1}{\sqrt{2 \pi}} \int_{-\infty}^{t} e^{-y^{2} / 2} d y, \quad \beta_{k}=\frac{\alpha_{k}}{d^{k}}, \quad \alpha_{k}=\mathbf{E} X_{1}^{k}, \\
J(t)=\frac{t(1-\Phi(t))}{\Phi^{\prime}(t)}=t e^{t^{2} / 2} \int_{t}^{\infty} e^{-y^{2} / 2} d y, \quad t \geqslant 0, \\
I(B)= \begin{cases}1, & \omega \in B, \\
0, & \omega \notin B,\end{cases} \\
m(z)=\frac{\varphi_{2 s+1}^{\prime}(z)}{\varphi_{2 s}(z)}, \quad \sigma^{2}(z)=\frac{\varphi_{2 s+2}^{\prime \prime}(z)}{\varphi_{2 s}(z)}-\left(\frac{\varphi_{2 s+1}^{\prime}(z)}{\varphi_{2 s}(z)}\right)^{2}, \quad \varphi_{s}(\lambda)=\sum_{0}^{s} \alpha_{k} \frac{\lambda^{k}}{k !}
\end{gathered}
$$

(имеются в виду производные по $z$ ). Всюду ниже через $\theta$ и $\delta$, с индексом или без него, будем обозначать разные величины, но такие, что $|\theta| \leqslant 1$, a $0 \leqslant \delta \leqslant 1$. Также пусть $\bar{B}$ всегда обозначает дополнение события $B$.

Подчеркнем следующее. Во-первых, все введенные величины $\zeta, x$, $\sigma^{2}, \nu, H(t)$ являются функциями $\lambda$, как $\theta$ и другие величины ниже. Однако чаще мы этого указывать не будем. Во-вторых, ранее в [1] через $m$ мы обозначали сумму математических ожиданий $m_{l}$, а не каждое из них, как сейчас. Наконец, в-третьих, различие преобразований Крамера (ПК), используемых в [1] и здесь, состоит в следующем. Величины $Y_{l}$ фактически определяются аналогично, но только в [1] функция $g_{\lambda}(t)=e^{\lambda t}$, а величины $X_{l}$ произвольны. Кроме того, ПК из [1] обладают свойством линейности, т.е. ПК линейной комбинации с.в. является соответствующей линейной комбинацией ПК данных с.в. (см. лемму 3 
из [2]). Здесь же это свойство отсутствует, и потому для записи искомой вероятности нужно непосредственно вводить преобразование (4) и с.в. $\eta$.

1.2. Каноническое представление. Рассматриваемые ниже характеристики всех с.в. являются функциями основного параметра $\lambda$. И прежде всего математическое ожидание и дисперсия с.в. $\zeta$. Поскольку дальнейшее во многом зависит от них, приведем не только соответствуюшие выражения (из них ясно, что сушествование указанных величин определяется условием (2)), но и краткий комментарий.

Так, связь параметров $x$ и $\lambda$ задается равенством (см. также п. 4)

$$
\begin{aligned}
& x=x(\lambda)=\mathbf{E} \zeta=n m=\frac{n}{s_{n}} m(z), \\
& \sigma^{2}=\sigma_{n}^{2}(\lambda)=\mathbf{D} \zeta=\frac{n}{s_{n}^{2}} \sigma^{2}(z)>0
\end{aligned}
$$

в силу (1). И это по-прежнему (как и в [1]) говорит о том, что уравнение $x=x(\lambda)$ имеет единственное решение $\lambda$ при всех возможных $x$ в широком диапазоне предположений. Правда, на этот раз $\mathbf{D} \zeta \neq x^{\prime}(\lambda)$, и потому сразу такой вывод, по-видимому, не следует. Однако эти функции «почти» равны асимптотически, поскольку теперь

$$
\sigma_{n}^{2}(\lambda)=x_{\lambda}^{\prime}(\lambda)+\frac{z^{2 s}\left[\alpha_{2 s+2}-m(z) \alpha_{2 s+1}\right]}{(2 s) ! \varphi_{2 s}(z) d^{2}}
$$

и второе слагаемое здесь является 0 -малым порядка $z^{2 s}$ при $z \rightarrow 0$. Более того, в условиях нашей теоремы даже оказывается, как и в [1], что $x(\lambda) \sim \lambda, \sigma^{2} \sim 1, n \rightarrow \infty$.

Покажем теперь, что справедлива следующая лемма.

Лемма 1. Если выполнень условия (1), (2), то при всех $\lambda>0 u$ соответствующих им $x$ из (5) имеет место представление

$$
\mathbf{P}\left\{S_{n}>s_{n} x\right\}=\varphi_{2 s}^{n}(z) \int_{0}^{\infty} \mathbf{E}(\eta \mid H=t) d H(t) .
$$

Д о к а з а т е л ь с т в о. Из независимости с.в. $\left(X_{l}\right)$ и $\left(Y_{l}\right)$ вытекает, что

$$
d \mathbf{P}\left\{S_{n}<s_{n} t\right\}=\int_{=t} \prod_{1}^{n} d \mathbf{P}\left\{X_{l}<s_{n} y_{l}\right\}, \quad d H(t)=\int_{=t} \prod_{1}^{n} d \mathbf{P}\left\{\bar{Y}_{l}<y_{l}\right\} ;
$$

символ $\int$ здесь и ниже использован для обозначения интегрирования по пространству $\mathbf{R}^{n}$ значений векторов $X / s_{n}, Y$ и $\bar{Y}=\left(\bar{Y}_{1}, \ldots, \bar{Y}_{n}\right)$, а символ « $=t \gg$ обозначает гиперплоскость $\sum_{1}^{n} y_{l}=t$. Таким образом, используя ОПК (4) и проводя элементарные выкладки, можно записать

$$
\begin{aligned}
\mathbf{P}\left\{S_{n}>s_{n} x\right\} & =\int_{x}^{\infty} d \mathbf{P}\left\{S_{n}<s_{n} t\right\}=\int_{x}^{\infty} \int_{=t} \prod_{1}^{n} d \mathbf{P}\left\{X_{l}<s_{n} y_{l}\right\} \\
& =\varphi_{2 s}^{n}(z) \int_{x}^{\infty} \int_{=t} \prod_{1}^{n} \frac{d \mathbf{P}\left\{Y_{l}<y_{l}\right\}}{e_{2 s}\left(\lambda y_{l}\right)}
\end{aligned}
$$




$$
\begin{aligned}
& =\varphi_{2 s}^{n}(z) \int_{0}^{\infty}\left\{\int_{=t} \prod_{1}^{n} \frac{d \mathbf{P}\left\{\bar{Y}_{l}<y_{l}\right\}}{e_{2 s}\left(\lambda\left(m+\sigma y_{l}\right)\right)}\left(\int_{=t} \prod_{1}^{n} d \mathbf{P}\left\{\bar{Y}_{l}<y_{l}\right\}\right)^{-1}\right\} d H(t) \\
& =\varphi_{2 s}^{n}(z) \int_{0}^{\infty} \mathbf{E}(\eta \mid H=t) d H(t)
\end{aligned}
$$

поскольку $\eta=\prod_{l=1}^{n} e_{2 s}^{-1}\left(\lambda\left(m+\sigma \bar{Y}_{l}\right)\right)$. Лемма доказана.

3 а м е ч а н и е 1 . При некоторых значениях $t$ условное математическое ожидание $\mathbf{E}=\mathbf{E}(\eta \mid H=t)$ может и не существовать. Однако на величине самого интеграла в (7) это не скажется, поскольку неопределенность будет уничтожена соответствующим нулевым элементом $d H(t)$. И все же часто в таких случаях полагают, скажем, что $\mathbf{E}=1$.

2. Одинаковые слагаемые. Основной результат. Из доказанной леммы следует, что область задания искомого представления на оси $\lambda$ представляет собой полуось, а на оси $x$ зависит от выражения (5). Однако нам желательно, прежде всего, получить такое представление, которое можно было бы сравнивать с аналогичным представлением при условии Крамера. В связи с этим рассмотрим на оси $\lambda$ интервал

$$
0<\lambda<C(\sqrt{n})^{1-\alpha}
$$

с произвольной постоянной $C$ и параметром $\alpha$, выбранным из условия

$$
\text { 1) } \alpha_{2 s+1}=0: \alpha>\frac{1}{s+1} ; \quad \text { 2) } \alpha_{2 s+1} \neq 0: \alpha>\frac{2}{2 s+1} \text {. }
$$

Обоснование нашего утверждения потребует также обозначений

$$
K_{n}(\varepsilon)=\left\{y \in \mathbf{R}^{n}:\left|y_{l}\right| \leqslant \varepsilon, 1 \leqslant l \leqslant n\right\}, \quad \varepsilon=(\sqrt{n})^{-\beta}, \quad A=\left\{\bar{Y} \in K_{n}(\varepsilon)\right\}
$$

для уменьшающегося куба $K_{n}(\varepsilon)$ пространства $\mathbf{R}^{n}$, поглощающего вектор $\bar{Y}$ с ростом $n$ в том смысле, что $\mathrm{P}(A) \rightarrow 1, n \rightarrow \infty$, если «скорость» $\beta$ его уменьшения не слишком велика. Кроме того, введем и обозначение $H_{(a, b)}$ для события $\{a \leqslant H<b\}, 0 \leqslant a \leqslant b \leqslant \infty\left(H_{t}=\{H=t\}\right)$.

Теорема. Если выполнень условия (1), (2), то для всех $\lambda$ из (9) и соответствуюших им $x$ из (5) при любых фиксированных $n \geqslant 1, T>0$ и а из (10) справедливо представление

$$
\mathbf{P}\left\{S_{n}>s_{n} x\right\}=\frac{\varphi_{2 s}^{n}(z) e^{-\lambda x}}{c \sigma(z) \sqrt{2 \pi}}\left(1+\delta_{n}(\lambda)\right),
$$

в котором

$$
\begin{gathered}
\delta_{n}(\lambda)=c \sigma(z) \sqrt{2 \pi}\left\{2 \theta \nu e^{\mu_{1}}+\Delta_{n}\right\}-\left\{1-\delta_{1} e^{\mu_{1}} J(c \sigma(z))\right\}, \\
\Delta_{n}=\delta_{2} e^{-\lambda \sigma T+\mu_{2}} \mathbf{P}\left(H_{[T, \infty)}\right)+\delta_{3} e^{\mu_{3}} \mathbf{P}(\bar{A}), \\
\delta_{1}=1-\frac{1-\Phi(\lambda \sigma+T)}{1-\Phi(\lambda \sigma)}, \quad 0<\delta_{2}, \delta_{3} \leqslant 1, \quad c=\frac{\lambda}{d}, \quad z=\frac{\lambda}{s_{n}},
\end{gathered}
$$

величины $\mu_{l}=\mu_{l}(n ; T), l=1,2, \mu_{3}=\mu_{3}(n)$ определень в (16), (17) ниже; при этом $\mu_{1} \rightarrow 0, \mu_{2} \rightarrow 0$, a $\mu_{3}$ ограничено при $n \rightarrow \infty, a \lambda \sigma=c \sigma(z)$. 
Д о к а з а т е л ь с т в о. В работе приведено полное и замкнутое обоснование этого результата. Оно весьма громоздко, и потому естественно объяснение всех его основных узлов отделить и поместить в пп. $3-5$, а здесь привести лишь схему доказательства. Разобьем ее на три части.

1. Вспомогательное ПБУ. Интеграл в правой части (7) совпадает с величиной $\mathbf{E}(\eta I(H \geqslant 0))$. Но для него можно получить и несколько иное представление $\left(I_{A}=I(A)\right)$

$$
\begin{aligned}
& \int_{0}^{\infty} \mathbf{E}\left(\eta \mid H_{t}\right) d H(t)=\int_{0}^{T} \mathbf{E}\left(\eta I_{A} \mid H_{t}\right) d H(t) \\
& \quad+\mathbf{E}\left(\eta I_{A} \mid H_{[T, \infty)}\right) \mathbf{P}\left(H_{[T, \infty)}\right)+\delta_{3} e^{-\lambda x+\mu} \mathbf{E}(\rho \mid \bar{A}) \mathbf{P}(\bar{A}) .
\end{aligned}
$$

Для этого достаточно разложить с.в. $\eta I(H \geqslant 0)$ в сумму трех с.в. соответственно разбиению события $\{H \geqslant 0\}=H_{[0, \infty)}$ на три подмножества

$$
H_{[0, \infty)}=A \cap H_{[0, T)}+A \cap H_{[T, \infty)}+\bar{A} \cap H_{[0, \infty)},
$$

ввести еще одну вспомогательную с.в.

$$
\rho=\prod_{1}^{n} \frac{e_{2 s}(\lambda m)}{e_{2 s}\left(\lambda\left(m+\sigma \bar{Y}_{l}\right)\right)}=\prod_{1}^{n} \frac{e_{2 s}(\lambda m)}{e_{2 s}\left(\lambda Y_{l}\right)}=e^{\lambda x-\mu} \eta
$$

( $\mu \rightarrow 0$ в силу свойства 2.4 ниже) и воспользоваться равенствами

$$
\begin{aligned}
\mathbf{E}\left(\eta I\left(A \cap H_{[0, T)}\right)\right) & =\int_{0}^{T} \mathbf{E}\left(\eta I_{A} \mid H_{t}\right) d H(t), \\
\mathbf{E}\left(\eta I\left(\bar{A} \cap H_{[0, \infty)}\right)\right) & =e^{-\lambda x+\mu} \mathbf{E}\left(\rho I\left(\bar{A} \cap H_{[0, \infty)}\right)\right)=\delta_{3} e^{-\lambda x+\mu} \mathbf{E}(\rho \mid \bar{A}) \mathbf{P}(\bar{A}) .
\end{aligned}
$$

2. Узловые факты, установленные ниже. Обоснование теоремы непосредственно опирается на ряд фактов разного уровня сложности, которым ниже мы присвоили номера 2.1-2.5. Условия справедливости всех этих свойств уточняются ниже, в пп. $3-5$, т.е. там, где они и доказываются. Соотношения 2.2 используются для записи в нужной форме первого, интегрального слагаемого в (15). При выяснении роли остальных двух слагаемых в этом представлении используются, наряду с 2.2 , последние соотношения 2.3-2.5:

$2.1 \mathbf{P}(A) \rightarrow 1, n \rightarrow \infty$;

$2.2 \quad \mathbf{E}\left(\eta I_{A} \mid H_{t}\right)=e^{-\lambda(x+\sigma t)} \mathbf{E}\left(\varrho I_{A} \mid H_{t}\right), \varrho=\exp \left(\sum_{1}^{n} c_{l}\left(\lambda Y_{l}\right)^{2 s+1}\right)$,

$$
\begin{aligned}
& e_{2 s}^{-1}(\lambda(m+\sigma \varepsilon))<(2 s+1) ! c_{l}<e_{2 s}^{-1}(\lambda(m-\sigma \varepsilon)) ; \\
& \mathbf{E}\left(\eta I_{A} \mid H_{[T, \infty)}\right)=\delta e^{-\lambda(x+\sigma T)} \mathbf{E}\left(\varrho I_{A} \mid H_{[T, \infty)}\right) ;
\end{aligned}
$$

$2.3 \mathbf{E}\left(\varrho I_{A} \mid H_{t}\right) \longrightarrow 1, n \rightarrow \infty$, равномерно по $0 \leqslant t \leqslant T$,

$$
\mathbf{E}\left(\varrho I_{A} \mid H_{(T, \infty)}\right) \longrightarrow 1, \quad n \rightarrow \infty
$$

$2.4 e_{2 s}^{n}(\lambda m) \sim e^{\lambda x}, \quad n \rightarrow \infty$;

$2.5 \limsup _{n \rightarrow \infty} \mathrm{E}(\rho \mid \bar{A}) \leqslant g_{s}<\infty$. 
В заключение все же уточним, где эти узловые моменты теоремы обоснованы. Так, свойства 2.2 и 2.4 устанавливаются в следствии 1 (п. 3), свойство 2.1 - в лемме 3 (п. 4), свойство 2.5 - в следствии 3 (п. 5). Что же касается свойств 2.3, то их элементарное доказательство (но при более жестком условии, чем (10)) приведено в лемме 6 , а основной вариант излагается в следствии 5, причем оба утверждения находятся В пा. 5 .

3. Формирование представления и ошибки $\delta_{n}(\lambda)$. Теперь чтобы получить искомое представление, фактически достаточно определить величины $\mu_{l}$. Сделаем это с помощью равенств

$$
\begin{aligned}
e^{\mu_{1}} \int_{0}^{T} e^{-\lambda \sigma t} d H(t) & =\int_{0}^{T} e^{-\lambda \sigma t} \mathbf{E}\left(\varrho I_{A} \mid H_{t}\right) d H(t), \\
\mathbf{E}\left(\varrho I_{A} \mid H_{[T, \infty)}\right) & =e^{\mu_{2}}, \quad e^{\mu} \mathbf{E}(\rho \mid \bar{A})=e^{\mu_{3}}
\end{aligned}
$$

и покажем, что представление (11) с элементами (12)-(14) действительно легко вытекает из леммы 1 и соотношения (15) в силу 2.1-2.5.

Прежде всего, ясно, что $\mu_{l} \rightarrow 0$ при $n \rightarrow \infty$ и $l=1,2$ в силу 2.3 , а ограниченность величин $\mu_{3}=\mu_{3}(n)$ при $n \rightarrow \infty$ вытекает из 2.5 и того, что $\mu \rightarrow 0$ в силу 2.4. Далее, нетрудно видеть, что во всех трех слагаемых в (15) появится множитель $e^{-\lambda x}$, если воспользоваться свойствами 2.2 и выражением $\eta=e^{\mu-\lambda x} \rho$. Причем без этого множителя второе и третье слагаемые можно записать в виде (13). Наконец, первому слагаемому можно придать форму $I_{1} e^{-\lambda x+\mu_{1}}$, где

$$
I_{1}=\int_{0}^{T} e^{-\lambda \sigma t} d H(t)=\frac{J(\lambda \sigma)}{\lambda \sigma \sqrt{2 \pi}} \delta_{1}+2 \theta \nu,
$$

если по аналогии с [2] (см. (9), (10)) воспользоваться представлением

$$
\begin{aligned}
& \int_{0}^{T} e^{-a t} d H(t)=\int_{0}^{T} e^{-a t} d \Phi(t)+\int_{0}^{T} e^{-a t} d(H(t)-\Phi(t)) \\
& =e^{a^{2} / 2}(1-\Phi(a)) \delta_{1}+\left.e^{-a t}(H(t)-\Phi(t))\right|_{0} ^{T}-\int_{0}^{T}(H(t)-\Phi(t)) d e^{-a t},
\end{aligned}
$$

в котором $a=\lambda \sigma=c \sigma(z)$. Теорема доказана.

3. Свойства усеченной экспоненты. Прежде чем интересоваться выводом искомого представления, естественно внести ясность в некоторые возникающие при его обосновании проблемы. Начнем с того, что укажем ряд свойств функции

$$
e_{2 s}(t)=1+t+\frac{t^{2}}{2}+\cdots+\frac{t^{2 s}}{(2 s) !},
$$

которые нам потребуются в дальнейшем. Как известно (см. [3, гл. II, c. 39,40$]$, где она обозначается $\left.\exp _{2 s}(t)\right)$, эта функция при всех $t \in \mathbf{R}$ 
положительна, и именно это позволяет использовать ее для построения OПK (4).

Функция $e_{2 s}(t)$ не только положительна, но и выпукла вниз при всех $t$, поскольку ее вторая производная $e_{2 s-2}(t)$ также положительна всюду. Поэтому функция $g(t)=e_{2 s}^{-1}(t)$ напоминает одновершинную плотность. Ее первая производная лишь раз меняет свой знак, а для нулей второй производной имеем уравнение $2 e_{2 s-1}^{2}=e_{2 s-2} e_{2 s}$. Поэтому $g(t)$ имеет одну точку максимума $t_{\mathrm{m}}(s)$ и две точки перегиба $t_{\mathrm{c}}^{-}(s)<t_{\mathrm{m}}(s)<t_{\mathrm{c}}^{+}(s)<0$. Иными словами, в интервале $t_{\mathrm{c}}^{-}(s)<t<t_{\mathrm{c}}^{+}(s)$ функция $g(t)$ выпукла вверх, а вне его - вниз. Ясно также, что

$$
\begin{gathered}
t_{\mathrm{c}}^{-}(1)=-1-\frac{1}{\sqrt{3}}<t_{\mathrm{m}}(1)=-1<t_{\mathrm{c}}^{+}(1)=-1+\frac{1}{\sqrt{3}} \\
g_{s} \equiv g\left(t_{\mathrm{m}}(s)\right)=(2 s) !\left(t_{\mathrm{m}}(s)\right)^{-2 s} .
\end{gathered}
$$

Но, возможно, главной точкой опоры при обосновании ПБУ без условия Крамера является тот факт, что в окрестности нуля усеченная и обычная экспоненты почти совпадают. Причем слово «почти» можно использовать тем с большим основанием, чем больше $s$. Так говорить позволяет следующее утверждение.

Лемма 2. При любых $y \in \mathbf{R}$

$$
\ln e_{2 s}(y)=y-A_{s}(y), \quad \text { əде } \quad A_{s}(y)=\int_{0}^{y} \frac{t^{2 s}}{(2 s) ! e_{2 s}(t)} d t .
$$

$B$ частности, при любьх $c \geqslant 0, k=2 s+1$ и указанньх ниже $y$

$$
\begin{aligned}
\frac{1}{e_{2 s}(y)} & =\exp \left(-y+a \frac{y^{k}}{k !}\right) \\
\text { 2) } \frac{e_{2 s}(c)}{e_{2 s}(c+y)} & =\exp \left(-y+b \frac{(c+y)^{k}-c^{k}}{k !}\right) ;
\end{aligned}
$$

при этом величины $a=a(y), b=b(c, y)$ удовлетворяют неравенствам

1) $y>0: e^{-y}<e_{2 s}^{-1}(y)<a<1 ; \quad t_{\mathrm{m}}(s)<y<0: 1<a<e_{2 s}^{-1}(y)<g_{s}$;

2) $y>0: e^{-(c+y)}<b<e_{2 s}^{-1}(c) ; \quad t_{\mathrm{m}}(s)-c<y<0: \quad e_{2 s}^{-1}(c)<b<g_{s}$.

Доказ ат ельство. Таккак $\left(\ln e_{2 s}(y)\right)_{y}^{\prime}=e_{2 s-1} / e_{2 s}=1-$ $y^{2 s} /\left((2 s) ! e_{2 s}(y)\right)$ и $\ln e_{2 s}(0)=0$, то $\ln e_{2 s}(y)=\int_{0}^{y}\left[1-t^{2 s} /\left((2 s) ! e_{2 s}(t)\right)\right] d t$. Поэтому основное соотношение (18) установлено. Приведенные же в лемме свойства 1), 2) вытекают из него и монотонности функции $e_{2 s}^{-1}(y)$ на интервале $y>t_{\mathrm{m}}(s)$. Лемма доказана.

Приведем сразу и пример практического использования этой леммы. Напомним: с.в. $\eta$ была введена в п. 1, а с.в. $\varrho$ и события $A, H_{t}-$ в п. 2. 
Следствие 1. При любых $0<t, T \leqslant n \varepsilon$ справедливы представления

$$
\begin{aligned}
& \mathbf{E}\left(\eta I_{A} \mid H_{t}\right)=e^{-\lambda(x+\sigma t)} \mathbf{E}\left(\varrho I_{A} \mid H_{t}\right) \quad\left(\text { с указаннылии } c_{l}\right) u \\
& \mathbf{E}\left(\eta I_{A} \mid H_{[T, \infty)}\right)=\delta e^{-\lambda(x+\sigma T)} \mathbf{E}\left(\varrho I_{A} \mid H_{[T, \infty)}\right),
\end{aligned}
$$

если условнье математические ожидания существуют $и \lambda(m-\sigma \varepsilon) \geqslant$ $t_{\mathrm{m}}(s)$.

Если же $(2 s+1) \alpha>1$, то равномерно по $\lambda$ из (9) имеем

$$
\prod_{1}^{n} e_{2 s}(\lambda m)=e_{2 s}^{n}(\lambda m) \sim e^{\lambda x}, \quad n \longrightarrow \infty .
$$

Доказат ельст в о. Если $y \in K_{n}(\varepsilon) \cap B_{t}, B_{t}=\left\{y \in \mathbf{R}^{n}\right.$ : $\left.\sum_{1}^{n} y_{l}=t\right\}$, то

$$
\prod_{1}^{n} \frac{1}{e_{2 s}\left(\lambda\left(m+\sigma y_{l}\right)\right)}=\exp \left\{-\lambda(x+\sigma t)+\sum_{1}^{n} a_{l} \frac{\left(\lambda\left(m+\sigma y_{l}\right)\right)^{2 s+1}}{(2 s+1) !}\right\}
$$

в силу п. 1 леммы 2. Нужно только учесть, что возможные значения с.в. $Y_{l}$ (т.е. $\left.m+\sigma y_{l}\right)$ представляют собой некоторую окрестность нуля и потому $e_{2 s}^{-1}(\lambda(m+\sigma \varepsilon)) \leqslant a_{l} \leqslant e_{2 s}^{-1}(\lambda(m-\sigma \varepsilon))$. Тем самым первое представление в 2.2 установлено. Чтобы обосновать второе, заметим, что событие $A \cap H_{t}$ пусто при $t>n \varepsilon$, т.е. $\mathbf{E}\left(\eta I_{A \cap H_{t}}\right)=0$. Поэтому

$$
\begin{gathered}
\mathbf{E}\left(\eta I\left(A \cap H_{[T, \infty)}\right)\right)=\int_{T}^{\infty} \mathbf{E}\left(\eta I_{A} \mid \overline{H_{t}}\right) d H(t)=\int_{T}^{n \varepsilon} \mathbf{E}\left(\eta I_{A} \mid H_{t}\right) d H(t) \\
\leqslant e^{-\lambda(x+\sigma T)} \int_{T}^{n \varepsilon} \mathbf{E}\left(\varrho I_{A} \mid H_{t}\right) d H(t)=e^{-\lambda(x+\sigma T)} \mathbf{E}\left(\varrho I\left(A \cap H_{[T, \infty)}\right)\right) .
\end{gathered}
$$

Наконец, тот же пункт леммы 2 позволяет сказать, что в равенстве $e_{2 s}^{n}(\lambda m)=\exp \left(n\left(\lambda m-a(\lambda m)^{k} / k !\right)\right) \sim \exp (\lambda x)$ параметр $a$ принимает значения в интервале $(0,1)$. Искомое же соотношение 2.4 , очевидно, имеет место, если $n(\lambda m)^{k} \rightarrow 0$. Иными словами, соотношение 2.4 имеет место, если $\lambda^{2 k} n^{1-k}=o(1)$, поскольку $m \sim \lambda / n$ (см. начало п. 4). Но для этого достаточно выбрать $\alpha$ из неравенства $(2 s+1) \alpha>1$. Следствие доказано.

4. Поглощающий куб. Асимптотика элементов. В этом пункте перед нами две цели. Во-первых, указать условия, при которых куб $K_{n}(\varepsilon)$ «поглошает» вектор $\bar{Y}$, т.е. равномерно по $\lambda$ из (9)

$$
2.1 p=\mathbf{P}(A)=\mathbf{P}\left\{\left|Y_{l}-m\right| \leqslant \sigma \varepsilon, 1 \leqslant l \leqslant n\right\} \longrightarrow 1, \quad n \rightarrow \infty .
$$

И, во-вторых, подготовить «почву» для обоснования соотношений 2.3 и 2.5. Для этого выясним условия «быстрого» убывания трех условных математических ожиданий. Но сначала уточним асимптотику основных элементов нашего ПБУ, которые в дальнейшем будут неоднократно 
использоваться. Для чего напомним, что все это нас интересует в ситуации, когда выполнены условия (1), (2), (9), а сторона куба определяется величиной $\varepsilon=(\sqrt{n})^{-\beta}$.

Итак, в указанных условиях при $n \rightarrow \infty$ (и $\alpha>0)$

$$
\begin{aligned}
\varphi_{2 s}(z) & =\sum_{0}^{2 s} \alpha_{k} \frac{z^{k}}{k !} \sim 1+\frac{d^{2} z^{2}}{2}=1+\frac{\lambda^{2}}{2 n}, \quad \varphi_{2 s+1}^{\prime}(z) \sim d^{2} z=d \frac{\lambda}{\sqrt{n}} \\
m(z) & =\frac{\sum_{1}^{2 s} \alpha_{k+1} z^{k} / k !}{\varphi_{2 s}(z)} \sim d^{2} z, \quad m=\frac{m(z)}{s_{n}} \sim \frac{\lambda}{n}, \quad x=\frac{n}{s_{n}} m(z) \sim \lambda \\
\sigma^{2}(z) & =\frac{\sum_{0}^{2 s} \alpha_{k+2} z^{k} / k !}{\varphi_{2 s}(z)}-m^{2}(z) \sim d^{2}\left[1+\beta_{3} \frac{\lambda}{\sqrt{n}}+\left(\beta_{4}-3\right) \frac{\lambda^{2}}{2 n}\right], \\
\sigma^{2} & =\frac{\sigma^{2}(z)}{d^{2}} .
\end{aligned}
$$

Покажем далее, что куб $K_{n}(\varepsilon)$ может быть поглошающим, если

$$
\alpha>(s+1)^{-1} \text {. }
$$

Используем для этого известное асимптотическое соотношение

$$
\int_{t}^{\infty} y^{k} d \mathbf{P}\left\{X_{l}<y\right\}=o\left(t^{-(2 s+2-k)}\right), \quad t \rightarrow \infty
$$

(и его аналог для интеграла $\int_{-\infty}^{t} y^{k} d \mathbf{P}\left\{X_{l}<y\right\}$ при $t \rightarrow-\infty$ ), справедливое при любом $0 \leqslant k \leqslant 2 s+2$, если выполнено условие (2).

Лемма 3. В условиях (1), (2), (9) соотношение (19) имеет место, если

$$
\beta<s(s+1)^{-1}, \quad \alpha+\beta \geqslant 1 .
$$

Д ок а з а т е л с т в о. При любом $0<\delta<1$ и достаточно больших $n$

$$
\begin{gathered}
1-p \leqslant \sum_{1}^{n} \int_{|t| \geqslant \varepsilon} d \mathbf{P}\left\{Y_{l}-m<\sigma t\right\} \leqslant \sum_{1}^{n} \int_{|t| \geqslant \delta \varepsilon} d \mathbf{P}\left\{Y_{l}<t\right\}, \\
\int_{|t| \geqslant \delta \varepsilon} d \mathbf{P}\left\{Y_{l}<t\right\}=\varphi_{2 s}^{-1}(z) \int_{|t| \geqslant \delta \varepsilon} e_{2 s}(\lambda t) d \mathbf{P}\left\{X_{l}<s_{n} t\right\} .
\end{gathered}
$$

Поэтому достаточно показать, что последний интеграл при всех $l$ есть $o\left(n^{-1}\right)$. Но это вытекает из условий, поскольку в силу (21) при любом $0 \leqslant k \leqslant 2 s$ :

$$
\begin{aligned}
& \int_{|t| \geqslant \delta \varepsilon}(\lambda t)^{k} d \mathbf{P}\left\{X_{l}<s_{n} t\right\}=\int_{|y| \geqslant \delta \varepsilon s_{n}}(z y)^{k} d \mathbf{P}\left\{X_{l}<y\right\} \\
& =z^{k} o\left(\left(\varepsilon s_{n}\right)^{-(2 s+2-k)}\right)=O\left((\sqrt{n})^{-(1-\beta)(2 s+2)+k(1-\alpha-\beta)}\right)
\end{aligned}
$$

Лемма доказана. 
Нетрудно видеть, что при $\alpha>1 /(s+1)$ условию $(22)$ удовлетворяет любое $\beta$ из интервала $[1-\alpha, s /(s+1))$. Таким образом, если выбрать параметр $\alpha$ из условия (20), то существуют скорости $\beta$ «убывания» куба $K_{n}(\varepsilon)$, при которых он «поглощает» вектор $\bar{Y}$ в смысле (19).

3 а м е ч а н и е 2. Отметим для сравнения, насколько быстро может уменьшаться куб $K_{n}(\varepsilon)$, в который с вероятностью 1 «заползает» вектор $X / s_{n}$ из одинаково нормально распределенных с.в. $X_{l}=N(0,1)$. Оказывается, что в этом случае можно даже положить $\varepsilon=((2 \ln n) / n)^{1 / 2}$. В самом деле, поскольку $s_{n}^{2}=n$ и $1-\Phi(x) \sim e^{-x^{2} / 2} /(x \sqrt{2 \pi}), x \rightarrow \infty$, то

$$
\mathbf{P}\left\{X s_{n}^{-1} \in K_{n}(\varepsilon)\right\}=(1-2(1-\Phi(\varepsilon \sqrt{n})))^{n} \longrightarrow 1, \quad n \rightarrow \infty .
$$

Перейдем теперь к решению второй задачи, т.е. к выявлению асимптотики трех условных математических ожиданий. Для этого сначала введем несколько новых обозначений: $t_{ \pm}=s_{n} y_{ \pm}, y_{ \pm}=m \pm \sigma \varepsilon$,

$$
\begin{aligned}
r_{m} & =\left(\int_{t_{+}}^{\infty}+\int_{-\infty}^{t_{-}}\right) t^{m} d \mathbf{P}\left\{X_{l}<t\right\}, \\
R_{m} & =\left(\int_{t_{+}}^{\infty}+\int_{-\infty}^{t_{-}}\right) t^{m} e_{2 s}(z t) d \mathbf{P}\left\{X_{l}<t\right\},
\end{aligned}
$$

и установим для этих математических ожиданий нужные представления.

Лемма 4. При выполнении условий (1), (2), (9) для любых $1 \leqslant l \leqslant n$ справедливы следующие представления:

$$
\begin{aligned}
& E_{1}=\mathbf{E}\left(\lambda \sigma \bar{Y}_{l}|| \bar{Y}_{l} \mid \leqslant \varepsilon\right)=z\left\{\frac{R_{0} \varphi_{2 s+1}^{\prime}(z)-R_{1} \varphi_{2 s}(z)}{\varphi_{2 s}(z)\left(\varphi_{2 s}(z)-R_{0}\right)}\right\}, \\
& E_{2}=\mathbf{E}\left(\left(\lambda Y_{l}\right)^{2 s+1}|| \bar{Y}_{l} \mid \leqslant \varepsilon\right)=\frac{I_{2}}{\varphi_{2 s}(z)-R_{0}}, \\
& E_{3}=\mathbf{E}\left(\left(\lambda Y_{l}\right)^{2(2 s+1)}|| \bar{Y}_{l} \mid \leqslant \varepsilon\right)=\frac{I_{3}}{\varphi_{2 s}(z)-R_{0}}
\end{aligned}
$$

при этом если $\alpha+\beta \geqslant 1$, то

$$
\begin{aligned}
& I_{2}=z^{2 s+1}\left(\alpha_{2 s+1}-r_{2 s+1}\right)+z^{2 s+2}\left(\alpha_{2 s+2}-r_{2 s+2}\right) O(1), \\
& I_{3}=z^{2 s+2}\left(\alpha_{2 s+2}-r_{2 s+2}\right) O(1), \quad n \rightarrow \infty .
\end{aligned}
$$

Док аз а тель с т в о. Поскольку $Y_{l}=m+\sigma \bar{Y}_{l}$, то в силу (3)

$$
\begin{aligned}
E_{1} & =\lambda \frac{\int_{y_{-}}^{y_{+}}(y-m) d \mathbf{P}\left\{Y_{l}<y\right\}}{\int_{y_{-}}^{y_{+}} d \mathbf{P}\left\{Y_{l}<y\right\}}=\lambda\left\{\frac{\int_{y_{-}}^{y_{+}} y e_{2 s}(\lambda y) d \mathbf{P}\left\{X_{l}<s_{n} y\right\}}{\int_{y_{-}}^{y_{+}} e_{2 s}(\lambda y) d \mathbf{P}\left\{X_{l}<s_{n} y\right\}}-m\right\} \\
& =z\left\{\frac{\int_{t_{-}}^{t_{+}} t e_{2 s}(z t) d \mathbf{P}\left\{X_{l}<t\right\}}{\int_{t_{-}}^{t_{+}} e_{2 s}(z t) d \mathbf{P}\left\{X_{l}<t\right\}}-m(z)\right\}=z\left\{\frac{\varphi_{k}^{\prime}(z)-R_{1}}{\varphi_{2 s}(z)-R_{0}}-\frac{\varphi_{k}^{\prime}(z)}{\varphi_{2 s}(z)}\right\},
\end{aligned}
$$




$$
\begin{aligned}
E_{2} & =\frac{\int_{y_{-}}^{y_{+}}(\lambda y)^{k} d \mathbf{P}\left\{Y_{l}<y\right\}}{\int_{y_{-}}^{y_{+}} d \mathbf{P}\left\{Y_{l}<y\right\}}=\frac{\int_{y_{-}}^{y_{+}}(\lambda y)^{k} e_{2 s}(\lambda y) d \mathbf{P}\left\{X_{l}<s_{n} y\right\}}{\int_{y_{-}}^{y_{+}} e_{2 s}(\lambda y) d \mathbf{P}\left\{X_{l}<s_{n} y\right\}} \\
& =\frac{\int_{t_{-}}^{t_{+}}(z t)^{k} e_{2 s}(z t) d \mathbf{P}\left\{X_{l}<t\right\}}{\int_{t_{-}}^{t_{+}} e_{2 s}(z t) d \mathbf{P}\left\{X_{l}<t\right\}}=\frac{I_{2}}{\varphi_{2 s}(z)-R_{0}}, \\
E_{3} & =\frac{\int_{y_{-}}^{y_{+}}(\lambda y)^{2 k} d \mathbf{P}\left\{Y_{l}<y\right\}}{\int_{y_{-}}^{y_{+}} d \mathbf{P}\left\{Y_{l}<y\right\}}=\frac{\int_{t_{-}}^{t_{+}}(z t)^{2 k} e_{2 s}(z t) d \mathbf{P}\left\{X_{l}<t\right\}}{\int_{t_{-}}^{t_{+}} e_{2 s}(z t) d \mathbf{P}\left\{X_{l}<t\right\}}=\frac{I_{3}}{\varphi_{2 s}(z)-R_{0}}
\end{aligned}
$$

$(k=2 s+1)$. Остается лишь объяснить, почему в последних равенствах для $E_{2}$ и $E_{3}$ числитель может быть записан указанным образом. Нетрудно видеть, однако, что в рассматриваемых условиях это вытекает из возможности представить подынтегральное выражение числителей в виде $(n \rightarrow \infty)$

$$
\begin{aligned}
& (z t)^{k} e_{2 s}(z t)=(z t)^{k}+(z t)^{2 s+2} \sum_{1}^{2 s} \frac{(z t)^{l-1}}{l !}=(z t)^{k}+(z t)^{2 s+2} O(1), \\
& (z t)^{2 k} e_{2 s}(z t)=(z t)^{2 s+2}\left[(z t)^{2 s} \sum_{0}^{2 s} \frac{(z t)^{l}}{l !}\right]=(z t)^{2 s+2} O(1),
\end{aligned}
$$

поскольку $\sup _{t_{-} \leqslant t \leqslant t_{+}} z t=\lambda(m+\sigma \varepsilon)=O(\lambda \varepsilon)=O(1)$. Лемма доказана.

Теперь, чтобы сформулировать и доказать искомые асимптотические соотношения, а заодно и объяснить, откуда берется экзотическое условие (10), нам остается лишь убедиться в том, что в силу (21) при $n \rightarrow \infty$

$$
\begin{aligned}
r_{m}=o\left((\sqrt{n})^{-(1-\beta)(2 s+2-m)}\right), & & 0 \leqslant m \leqslant 2 s+2, \\
R_{m}=o\left((\sqrt{n})^{-(1-\beta)(2 s+2-m)}\right), & & 0 \leqslant m \leqslant 2 .
\end{aligned}
$$

В самом деле, поскольку $t_{ \pm}=s_{n}(m \pm \sigma \varepsilon) \sim \pm s_{n} \varepsilon= \pm d(\sqrt{n})^{1-\beta}$, то $r_{m}=o\left(\left(s_{n} \varepsilon\right)^{-(2 s+2-m)}\right)=o\left((\sqrt{n})^{-(1-\beta)(2 s+2-m)}\right)$. Что же касается величины $R_{m}$, то достаточно заметить, скажем, что $\int_{t_{+}}^{\infty} z^{k} t^{m+k} d \mathbf{P}\left\{X_{l}<t\right\}=$ $z^{k} o\left(\left(s_{n} \varepsilon\right)^{-(2 s+2-m-k)}\right)=o\left((\sqrt{n})^{-k \alpha-(1-\beta)(2 s+2-m-k)}\right)$, если $m+k \leqslant 2 s+2$. Причем для последнего показателя неравенство $k(1-\alpha-\beta)-(1-\beta)(2 s+$ $2-m) \leqslant-(1-\beta)(2 s+2-m)$ справедливо при всех $0 \leqslant k \leqslant 2 s$ и ясно, что «все хорошо» при $m \leqslant 2$ (поскольку тогда $k \leqslant 2 s \Rightarrow m+k \leqslant 2 s+2$ ).

Следствие 2. В условиях (1), (2), (22) равномерно по $\lambda$ из (9)

$$
E_{1}=\mathbf{E}\left(\lambda \sigma \bar{Y}_{l}|| \bar{Y}_{l} \mid \leqslant \varepsilon\right)=o\left(n^{-1}\right), \quad n \rightarrow \infty .
$$

Если же выполнено и условие (10), то тажже при всех $1 \leqslant l \leqslant n$

$$
\begin{aligned}
& E_{2}=\mathbf{E}\left(\left(\lambda Y_{l}\right)^{2 s+1}|| \bar{Y}_{l} \mid \leqslant \varepsilon\right)=o\left(n^{-1}\right), \quad n \rightarrow \infty, \\
& E_{3}=\mathbf{E}\left(\left(\lambda Y_{l}\right)^{2(2 s+1)}|| \bar{Y}_{l} \mid \leqslant \varepsilon\right)=o\left(n^{-1}\right), \quad n \rightarrow \infty .
\end{aligned}
$$


Доказ а т ельст в о. В силу $(23) R_{0}=o\left((\sqrt{n})^{-(1-\beta)(2 s+2)}\right)$ и $R_{1}=o\left((\sqrt{n})^{-(1-\beta)(2 s+1)}\right)$, а $\varphi_{2 s}(z) \rightarrow 1, n \rightarrow \infty$, при $\alpha>0$. Поэтому $E_{1} \sim-z R_{1} \sim o\left((\sqrt{n})^{-\alpha-(1-\beta)(2 s+1)}\right)$. Для доказательства соотношения (24) остается заметить, что $-\alpha-(1-\beta)(2 s+1) \leqslant-(1-\beta)(2 s+2)<-2$ в силу (22).

Для доказательства же соотношения (25) достаточно показать, что

$$
I_{0} \equiv z^{2 s+1}\left(\alpha_{2 s+1}-r_{2 s+1}\right)+z^{2 s+2}\left(\alpha_{2 s+2}-r_{2 s+2}\right)=o\left(n^{-1}\right), \quad n \rightarrow \infty,
$$

если выполнено условие (10). Но это очевидно, так как при $\alpha_{2 s+1} \neq 0$ имеем $I_{0} \sim z^{2 s+1} \alpha_{2 s+1}=O\left((\sqrt{n})^{-\alpha(2 s+1)}\right)$, т.е. $I_{0}=o\left(n^{-1}\right)$, если $\alpha>2 /(2 s+1)$. Если же $\alpha_{2 s+1}=0$, то нетрудно показать, что $I_{0} \sim z^{2 s+2} \alpha_{2 s+2}=O\left((\sqrt{n})^{-\alpha(2 s+2)}\right)$ и, следовательно, $I_{0}=o\left(n^{-1}\right)$, если $\alpha>1 /(s+1)$. Наконец, равенство $E_{3}=o\left(n^{-1}\right)$ также вытекает из $(20)$. Следствие доказано.

5. Условные математические ожидания. Теперь мы готовы провести обоснование наиболее важного момента доказательства теоремы - асимптотического соотношения 2.5, а также оставшихся свойств 2.3.

5.1. Ограниченность математических ожиданий $\mathbf{E}(\rho \mid \bar{A})$, m.е. свойство 2.5. Сначала сформулируем и докажем один общий результат, а затем уже интересующий нас факт получим в следствии 3 как его частный случай. Предположим, что помимо последовательности н.о.p.с.в. $\xi=\left(\xi_{l}\right)_{l \geqslant 1}$ с $\mathbf{E} \xi_{1}=0, \mathbf{D} \xi_{1}=d^{2}$, определенных на одном и том же вероятностном пространстве $(\Omega, \mathscr{F}, \mathbf{P})$ и для которых, как и обычно, $S_{n}=\sum_{1}^{n} \xi_{l}, s_{n}^{2}=\mathrm{D} S_{n}$, заданы последовательность борелевских подмножеств $\left(A_{l}\right)_{l \geqslant 1}$ на прямой и последовательность одномерных борелевских функций $\left(f_{l}(t)\right)_{l \geqslant 1}$. Введем также обозначения:

$$
\begin{gathered}
\xi(n)=\left(\xi_{1}, \ldots, \xi_{n}\right), \quad y=\left(y_{1}, \ldots, y_{n}\right), \quad \mathscr{F}(y)=\prod_{1}^{n} f_{n}\left(y_{l}\right), \quad \rho=\mathscr{F}(\xi(n)), \\
A(n)=\left\{y \in \mathbf{R}^{n}: y_{l} \in A_{n}, 1 \leqslant l \leqslant n\right\}, \quad q=\mathbf{P}\left\{\xi_{1} \in A_{n}\right\}, \quad p=\mathbf{P}\left\{\xi_{1} \notin A_{n}\right\} .
\end{gathered}
$$

Лемма 5. Если последовательность множеств $\left(A_{l}\right)_{l \geqslant 1}$, функ-

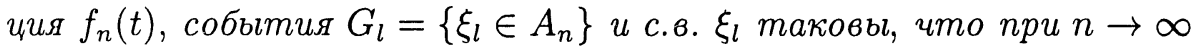

$$
\begin{aligned}
& \text { 1) } \left.p=o\left(n^{-1}\right), \quad 2\right) \quad \mathbf{E}\left(f_{n}\left(\xi_{l}\right) \mid G_{l}\right) \leqslant L_{n}=1+\frac{a_{n}}{n}, \quad t \in A_{n}, \\
& \text { 3) } f_{n}(t) \leqslant D_{n}, \quad t \notin A_{n},
\end{aligned}
$$

причем $\lim a_{n}=a, \lim D_{n}=D$, где а и $D$ - некоторье числа, то

$$
\limsup _{n \rightarrow \infty} \mathbf{E}(\rho \mid \xi(n) \notin A(n)) \leqslant D e^{a} .
$$


Д о к а з а т е л ь с т в о. Разобьем событие $B=\{\xi(n) \notin A(n)\}$ на $2^{n}-1$ непересекающихся подмножеств из $\mathscr{F}$ :

$$
B_{i j}=\left\{\xi_{l_{s}} \notin A_{n}, 1 \leqslant s \leqslant i ; \xi_{l} \in A_{n}, l \neq l_{s}\right\}, \quad 1 \leqslant i \leqslant n, \quad 1 \leqslant j \leqslant C_{n}^{i} .
$$

Тогда $\mathbf{P}\left(B_{i j}\right)=p^{i} q^{n-i}$ и ясно также, что $\mathbf{E}\left(f_{n}\left(\xi_{l}\right) \mid \bar{G}_{l}\right) \leqslant D_{n}$. Поэтому

$$
\begin{aligned}
\mathbf{E}\left(\rho I\left(B_{i j}\right)\right) & =\prod_{s=1}^{i} \mathbf{E}\left(f_{n}\left(\xi_{l_{s}}\right) I\left(\bar{G}_{l_{s}}\right)\right) \prod_{l \neq l_{s}} \mathbf{E}\left(f_{n}\left(\xi_{l}\right) I\left(G_{l}\right)\right) \leqslant\left(D_{n} p\right)^{i}\left(L_{n} q\right)^{n-i}, \\
\mathbf{E}(\rho I(B)) & \leqslant \sum_{1}^{n} C_{n}^{i}\left(D_{n} p\right)^{i}\left(L_{n} q\right)^{n-i}=\left(L_{n} q\right)^{n}\left[\left(1+\frac{D_{n} p}{L_{n} q}\right)^{n}-1\right] .
\end{aligned}
$$

Но $\mathbf{P}(B)=1-q^{n}=q^{n}\left[(1+p / q)^{n}-1\right]$ и если $\bar{p}=n p$, то

$$
\begin{aligned}
\mathbf{E}(\rho \mid B) & \leqslant \frac{L_{n}^{n}\left[\left(1+D_{n} p /\left(L_{n} q\right)\right)^{n}-1\right]}{(1+p / q)^{n}-1} \sim \frac{L_{n}^{n}\left(e^{D_{n} \bar{p} / L_{n} q}-1\right)}{e^{\bar{p} / q}-1} \\
& \sim L_{n}^{n}\left(\frac{D_{n}}{L_{n}}\right) \rightarrow D e^{a}, \quad n \rightarrow \infty
\end{aligned}
$$

поскольку $\bar{p}=o(1)$. Лемма доказана.

Следствие 3. В условиях (1), (2), (9), (22) выполнено соотношение

$$
2.5 \quad \limsup _{n \rightarrow \infty} \mathbf{E}(\rho \mid \bar{A}) \leqslant g_{s}=(2 s) !\left(t_{\mathrm{m}}(s)\right)^{-2 s} .
$$

Д ок а за т ельст в о. Лемма 5 остается в силе, если вместо последовательности с.в. $\left(\xi_{l}\right)_{l \geqslant 1}$ рассматривать схему серий н.о.р.с.в. $\left(\xi_{l n}\right)_{1 \leqslant l \leqslant n}$ при $n \geqslant 1$. Поэтому можно применить эту лемму к с.в. $\rho$ из (16), полагая $\xi_{l n}=\bar{Y}_{l}, f_{n}(t)=e_{2 s}(\lambda m) e_{2 s}^{-1}(\lambda(m+\sigma t)), A_{n}=\{y \in \mathbf{R}$ : $\left.|y| \leqslant \varepsilon=(\sqrt{n})^{-\beta}\right\}$, и, следовательно, событие $\bar{A}$ будет дополнением события $A=\left\{\bar{Y} \in K_{n}(\varepsilon)\right\}=\{\xi(n) \in A(n)\}$. Таким образом, для доказательства соотношения (27) достаточно убедиться в том, что условия (26) выполняются при $a=0, D=g_{s}$.

Мы знаем, что функция $e_{2 s}^{-1}(t)$ достигает максимума в точке $t_{\mathrm{m}}(s)$. Причем ее максимальное значение $g_{s}=(2 s) !\left(t_{\mathrm{m}}(s)\right)^{-2 s}$. Нетрудно видеть также, что максимальное значение функции $f_{n}(t)$ при $|t|>\varepsilon$ есть $D_{n}=e_{2 s}(\lambda m) g_{s} \longrightarrow g_{s}, n \rightarrow \infty$. Тем самым свойство (26.3) очевидно, как и (26.1), поскольку $p=\mathrm{P}\left\{\left|\bar{Y}_{l}\right|>\varepsilon\right\}=R_{0} \varphi_{2 s}^{-1}(z)=o\left(n^{-1}\right)$ при $\beta<s /(s+1)$.

Чтобы убедиться в справедливости оставшегося свойства (26.2), используем п. 2 леммы 2 , в соответствии с которым на множестве $\{|t| \leqslant \varepsilon\}$

$$
f_{n}(t)=\exp \left(-\lambda \sigma t+\frac{b\left[(\lambda(m+\sigma t))^{2 s+1}-(\lambda m)^{2 s+1}\right]}{(2 s+1) !}\right),
$$


причем можно считать, что $b=\exp (\lambda(m+\theta \sigma \varepsilon))$. Поэтому $(k=2 s+1)$

$$
\begin{aligned}
\mathbf{E}\left(f_{n}\left(\bar{Y}_{l}\right)|| \bar{Y}_{l} \mid \leqslant \varepsilon\right) & \sim \mathbf{E}\left(\exp \left(-\lambda \sigma \bar{Y}_{l}+\left(\lambda Y_{l}\right)^{k}(k !)^{-1}\right)|| \bar{Y}_{l} \mid \leqslant \varepsilon\right) \\
& \sim \mathbf{E}\left(\left[1-\lambda \sigma \bar{Y}_{l}+\left(\lambda Y_{l}\right)^{k}(k !)^{-1}\right]|| \bar{Y}_{l} \mid \leqslant \varepsilon\right) \\
& =1-E_{1}+E_{2}(k !)^{-1}=1+o\left(n^{-1}\right), \quad n \rightarrow \infty,
\end{aligned}
$$

если $\lambda \varepsilon=o(1)$, т.е. $\alpha+\beta>1$. Следствие доказано.

3 а м е ч а н и е 3 . Мы только что использовали условие $\alpha+\beta>1$ вместо второго неравенства из (22). Дело в том, что оба эти неравенства сами по себе не нужны, они играют вспомогательную роль при установлении асимптотики (19), а для ее справедливости достаточно и более сильного неравенства.

5.2. Сходимость условных математических ожиданий с.в. $I_{A}$, m.e. свойства 2.3. Начнем с того, что покажем: небольшое усиление

$$
\alpha>\frac{1}{s+1}+\frac{2}{2 s+1}
$$

условия (10) позволяет обосновать искомые свойства элементарно.

Лемма 6. Если выполнены условия (10а) и (22), то свойства 2.3 имеют место при сохранении остальных свойств.

Д о к а з а т е л ь с т в о. В разбиении события $H_{[0, \infty)}$ на три части из п. 2 при $T>n \varepsilon$ вторая часть исчезает, поскольку $A \cap H_{[T, \infty)}=\varnothing$. Поэтому достаточно установить лишь, что $\mathbf{E}\left(\varrho I_{A} \mid H_{t}\right) \rightarrow 1$ равномерно по $t, 0 \leqslant t \leqslant T \leqslant n \varepsilon$. Иными словами, достаточно убедиться в том, что $\sum_{1}^{n}\left(\lambda Y_{l}\right)^{2 s+1}=o(1)$ п.н. $(k=2 s+1)$, поскольку $a_{l} \sim 1 /(2 s+1) !, n \rightarrow \infty$.

Ho $\left|\lambda Y_{l}\right| \leqslant \lambda(m+\sigma \varepsilon) \sim \lambda \varepsilon=O\left((\sqrt{n})^{1-\alpha-\beta}\right), n \rightarrow \infty$. Поэтому $\sum_{1}^{n}\left(\lambda Y_{l}\right)^{2 s+1}=O\left((\sqrt{n})^{2+(2 s+1)(1-\alpha-\beta)}\right)=o(1)$, если $\alpha+\beta>1+2 /(2 s+1)$. Поскольку выполнено условие $(22)$, т.е. $\beta<s /(s+1)$, ясно, что нужное асимптотическое соотношение можно обеспечить при выборе (10a).

Условие (10а) естественно назвать небольшим усилением, поскольку по-прежнему можно будет считать, что $\alpha \rightarrow 0, s \rightarrow \infty$. Но, конечно, при этом формулировка теоремы будет относиться лишь к значениям $s \geqslant 2$, поскольку при $s=1$ значение $\alpha$ не сможет быть меньше 1 . А это нужно для того, чтобы интервал для $\lambda$ из (9) увеличивался при $n \rightarrow \infty$.

Укажем далее на соображение, которое может привести к одному из простейших доказательств искомых свойств при условии (10). Дело в том, что

$$
\mathbf{E}\left(\varrho I_{A} \mid H_{t}\right)=\mathbf{E}\left(\varrho \mid A \cap H_{t}\right) \mathbf{P}\left(A \mid H_{t}\right),
$$

а с другой стороны, вектор $\bar{Y}$ и с.в. $H$ асимптотически (при $n \rightarrow \infty$ ) независимы. Поэтому должны выполняться; скажем, соотношения

$$
\mathrm{E}\left(\varrho \mid A \cup H_{t}\right) \sim \mathrm{E}(\varrho \mid A), \quad \mathbf{P}\left(A \mid H_{t}\right) \sim \mathbf{P}(A), \quad n \rightarrow \infty .
$$


Поэтому искомые свойства будут вытекать из леммы 3 и следующего утверждения, фактически доказанного в следствии 2.

Следствие 4. В условиях (1), (2), (9), (10)

$$
\mathbf{E}(\varrho \mid A) \rightarrow 1, \quad n \rightarrow \infty .
$$

Д о к а з а т л ь с т в о. Достаточно заметить, что

$$
\mathbf{E}(\varrho \mid A)=\prod_{1}^{n} \mathbf{E}\left(\exp \left\{c_{l}\left(\lambda Y_{l}\right)^{2 s+1}\right\}|| \bar{Y}_{l} \mid \leqslant \varepsilon\right) .
$$

А теперь мы попробуем обосновать искомые свойства при условии (10), без использования асимптотической независимости вектора $\bar{Y}$ от с.в. $H$, т.е. понятия, которое и определить-то непросто из-за того, что число компонент этого вектора бесконечно растет, а опираясь на его «отчетливую» асимптотическую вырожденность и следствие 2 . Введем для этого последовательность серий н.о.р.с.в. $\left(\xi_{l n}\right)_{1 \leqslant l \leqslant n}, n \geqslant 1$, с распределением $\mathbf{P}\left\{\xi_{l n}<y\right\}=\mathbf{P}\left\{\left(\lambda Y_{l}\right)^{2 s+1}<y|| \bar{Y}_{l} \mid \leqslant \varepsilon\right\}$, и положим $\xi_{n}=\sum_{1}^{n} \xi_{l n}$. Можно сказать, что вспомогательная с.в. $\xi_{n}$ определена на меняюшемся вероятностном пространстве $\left(A, \mathscr{F}_{A}, \mathbf{P}_{A}\right)$, если исходная последовательность с.в. $\left(X_{l}\right)$ задана на пространстве $(\Omega, \mathscr{F}, \mathbf{P}), \mathscr{F}_{A}-$ сужение $\sigma$-алгебры $\mathscr{F}$ на множество $A$ и $\mathbf{P}_{A}(d \omega)=\mathbf{P}(d \omega) / \mathbf{P}(A)$. Иными словами, с.в. $\varrho$ на множестве $A$ принимает те же значения, что и с.в. $e^{a \xi_{n}}$, но только «с вероятностями, в $1 / \mathbf{P}(A)$ раз большими» ( $a$ - некоторая с.в. такая, что $a(2 s+1) ! \rightarrow 1$ п.н., $n \rightarrow \infty)$.

Следствие 5. В условиях (1), (2), (9), (10)

$2.3 \mathbf{E}\left(\varrho I_{A} \mid H_{t}\right) \longrightarrow 1, \quad n \rightarrow \infty$, равномерно по $0 \leqslant t \leqslant T \leqslant n \varepsilon ;$ $\mathbf{E}\left(\varrho I_{A} \mid H_{[T, \infty)}\right) \longrightarrow 1, \quad n \rightarrow \infty$.

Д о к а з а т ел в с т в о. Сначала покажем от противного, что

$$
e^{\xi_{n}} \rightarrow 1 \quad \text { п.н. при } n \rightarrow \infty .
$$

Пусть $\xi_{n} \nrightarrow 0$ п.н. Тогда $\mathbf{P}\left\{\liminf _{n} \xi_{n} \neq \limsup _{n} \xi_{n}\right\}>0$ (на самом деле в силу закона 0 и 1 эта вероятность равна единице!), и поскольку $\mathbf{E} \xi_{n}=n E_{2} \rightarrow 0$, то обязательно будет иметь место одно из следующих двух утверждений: либо $\mathbf{P}\left\{\liminf _{n} \xi_{n}<0, \limsup _{n} \xi_{n} \geqslant 0\right\}>0$, либо $\mathbf{P}\left\{\liminf \xi_{n} \leqslant 0, \limsup _{n} \xi_{n}>0\right\}>0$. Но в обоих случаях это войдет в противоречие с тем, что (см. следствие 2) $\mathbf{D} \xi_{n}=n\left(E_{3}-\left(E_{2}\right)^{2}\right) \sim n E_{3} \rightarrow 0$ при условии (20) и, тем более, при более жестком условии (10).

С другой стороны, если указанная сходимость имеет место, то тем более имеет место аналогичная сходимость для условных математических ожиданий вида $\mathbf{E}\left(e^{\xi_{n}} \mid \sigma_{n}\right), n \rightarrow \infty$, относительно произвольных 
$\sigma$-алгебр $\sigma_{n}$, более грубых, чем $\sigma$-алгебра, порожденная вектором $\bar{Y}$. Поэтому $\mathbf{E}\left(e^{a \xi_{n}} \mid H\right) \rightarrow 1$ п.н. и, в частности, при любом $t \leqslant T$

$$
\mathbf{E}\left(e^{a \xi_{n}} \mid H_{t}\right)=\frac{\mathbf{E}\left(\varrho I_{A} \mid H_{t}\right)}{\mathbf{P}(A)} \longrightarrow 1, \quad n \rightarrow \infty .
$$

Таким образом, первое свойство в 2.3 можно считать в силу 2.1 установленным при небольших и фиксированных $T$, поскольку равномерная сходимость для таких $T$ объясняется просто.

Возьмем произвольное разбиение отрезка $[0, T)$ из леммы 7 ниже и соответствующее ему разбиение множества элементарных исходов $B(m): \Omega=\sum_{0}^{m+1} B_{i}, B_{i}=\left\{H \in J_{l}(m)\right\}, 1 \leqslant l \leqslant m, B_{0}=\{H<0\}$, $B_{m+1}=\{H \geqslant T\}, B_{i} \cup B_{j}=\varnothing, i \neq j$. Тогда в силу доказанного ступенчатая функция $\mathbf{E}\left(e^{a \xi_{n}} \mid B(m)\right) \rightarrow 1$ п.н., а наша функция $\mathbf{E}\left(e^{a \xi_{n}} \mid H_{t}\right)$ на отрезке $[0, T)$ «лежит» между ее внутренними ступеньками, каждая из которых «подтягивается» к 1 при $n \rightarrow \infty$. При этом для небольших $T$ скорость сходимости к 1 у разных ступенек отличается немного. Чтобы обосновать второе свойство, достаточно в качестве $\sigma$-алгебры $\sigma_{n}$ взять порождаемую и вовсе простым разбиением $B(1)$.

6. Условная вероятность. В силу (28) при получении основного, первого элемента представления теоремы (из трех в (15)) потребуется вычислять условную вероятность $\mathbf{P}\left(A \mid H_{t}\right)$ как функцию параметра $t$. Приведем некоторые соображения, позволяющие находить либо сами эти функции, либо оценки для них снизу, а также дадим пример такой функции. При этом для простоты, но не снижая общности, будем рассматривать отвлеченную ситуацию, т.е. интересоваться условной вероятностью вида

$$
p_{n}(\varepsilon ; t)=\mathbf{P}\left\{\frac{\left|\xi_{l}\right|}{s_{n}} \leqslant \varepsilon, 1 \leqslant l \leqslant n \mid \frac{1}{s_{n}} \sum_{1}^{n} \xi_{l}=t\right\},
$$

где $\left(\xi_{l}\right)_{l \geqslant 1}$ - произвольная последовательность н.о.р.с.в. с $\mathbf{E} \xi_{1}=0$, $\mathrm{D} \xi_{1}=d^{2}$, полагая, как и обычно, $S_{n}=\sum_{1}^{n} \xi_{l}, s_{n}^{2}=\mathrm{D} S_{n}$, - хотя интересуюшая нас вероятность $\mathbf{P}\left(A \mid H_{t}\right)$ явно касается схемы серий, т.е. $\bar{Y}_{l}=\xi_{l n}$.

6.1. Оченка снизу. Чтобы сформулировать и доказать следующее утверждение, введем еще несколько обозначений:

$$
\begin{gathered}
A=\left\{\omega \in \Omega: s_{n}^{-1}\left|\xi_{l}\right| \leqslant \varepsilon, 1 \leqslant l \leqslant n\right\}, \quad B=B_{l m}(n)=\left\{\omega: s_{n}^{-1} S_{n} \in J_{l}(m)\right\}, \\
J_{l}(m)=\left(t_{l-1}, t_{l}\right], \quad t_{l}=t_{l}(m)=T l m^{-1}, \\
0<T<\infty, \quad m \geqslant 1, \quad 1 \leqslant l \leqslant m, \\
\Phi_{l m}=\Phi\left(t_{l}\right)-\Phi\left(t_{l-1}\right), \quad \nu_{n}=\sup _{t}\left|\mathscr{F}_{n}(t)-\Phi(t)\right|, \quad \mathscr{F}_{n}(t)=\mathbf{P}\left\{S_{n}<s_{n} t\right\} .
\end{gathered}
$$




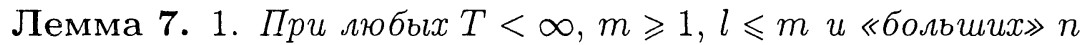

$$
\mathbf{P}\left(A \mid B_{l m}(n)\right) \geqslant \mathbf{P}(A)-\frac{\gamma_{n}}{\Phi_{l m}-2 \nu_{n}}, \quad \gamma_{n}=1-\mathbf{P}(A) .
$$

2. Cтупенчатая функиия $\sum_{l=1}^{m} \mathbf{P}\left(A \mid B_{l m}(n)\right) I\left(t \in J_{l}(m)\right)$ является усреднением плотности $p_{n}(\varepsilon ; t)$ на всех интервалах нашего разбиения отрезка $[0, T), T \leqslant n \varepsilon$. Поэтому если функция $p_{n}(\varepsilon ; t)$ монотонно убывает при $t \geqslant 0$, то для нее можно получить простую оченку снизу.

Д о к а з а т ел ь с т в о. 1. В силу теоремы Колмогорова (см. [4, гл. II, §9, п. 1]) можно считать, что на вероятностном пространстве $(\Omega, \mathscr{F}, \mathbf{P})$ одновременно заданы не только с.в. $\left(\xi_{l}\right)_{1 \leqslant l \leqslant \infty}$, но и независимые от них и так же распределенные с.в. $\left(\tilde{\xi}_{l}\right)_{1 \leqslant l \leqslant \infty}$. Поэтому можно ввести события $\widetilde{A}$, построенные по с.в. $\tilde{\xi}_{l}$ по аналогии с $A$. Тогда нетрудно видеть, что $\mathbf{P}(\widetilde{A})=\mathbf{P}(A)$ и, кроме того,

$$
|\mathbf{P}(\widetilde{A} B)-\mathbf{P}(A B)| \leqslant \max (\mathbf{P}((\widetilde{A} \backslash A) B), \mathbf{P}((A \backslash \widetilde{A}) B)) \leqslant \min \left(\gamma_{n}, \mathbf{P}(B)\right) .
$$

Если же и $\gamma_{n}<\mathbf{P}(B)$, то

$$
\mathbf{P}(A \mid B)=\frac{\mathbf{P}(A B)}{\mathbf{P}(B)}=\frac{\mathbf{P}(\widetilde{A} B)-[\mathbf{P}(\widetilde{A} B)-\mathbf{P}(A B)]}{\mathbf{P}(B)} \geqslant \mathbf{P}(A)-\frac{\gamma_{n}}{\mathbf{P}(B)}
$$

Остается заметить, что $\mathbf{P}\left(B_{l m}(n)\right)=\Phi_{l m}+2 \theta \nu_{n}$.

2. Нетрудно видеть, что на любом элементе разбиения

$$
\mathbf{P}\left(A \mid B_{l m}(n)\right)=\frac{\int_{t_{l-1}}^{t_{l}} p_{n}(\varepsilon ; t) d \mathbf{P}\left\{S_{n}<s_{n} t\right\}}{\mathbf{P}\left(B_{l m}(n)\right)} .
$$

Тем самым, действительно, указанная ступенчатая функция является усреднением нашей плотности, и потому для получения оценки снизу остается лишь использовать оценку из п. 1. Лемма доказана.

6.2. Рекуррентные соотношения. Представляется целесообразным сначала установить рекуррентные соотношения для частного случая стандартно нормально распределенных с.в. $\xi_{l} \equiv N(0,1)$, а уже затем получить искомые рекуррентные соотношения для произвольных $\xi_{l}$ как простое следствие. Тем более, что рекуррентные соотношения в нормальном случае полезны по разным причинам с практической точки зрения.

Лемма 8. $E$ сли $d \mathbf{P}\left\{\xi_{1}<t\right\}=\phi(t) d t, \phi(t)=(1 / \sqrt{2 \pi}) \exp \left(-t^{2} / 2\right), m o$

$$
p_{n}(\varepsilon ; t)=\int_{a_{-}}^{a_{+}} p_{n-1}\left(\varepsilon c_{n} ; \frac{t}{c_{n}}-y\right) s_{n} \phi\left(s_{n} y\right) d y, \quad|t| \leqslant n \varepsilon \quad(n \geqslant 2) \text {, }
$$

u $p_{1}(\varepsilon ; t)=I(|t| \leqslant \varepsilon) ;$ здесь $a_{ \pm}=( \pm \varepsilon n-t) /\left(s_{n} s_{n-1}\right), c_{n}=s_{n} / s_{n-1}, I(A)-$ индикаторная функиия множества $A, s_{n}^{2}=n$. 
Д ок аз а т ельст в о. Положим $A=K_{n}(\varepsilon)=\left\{\omega:\left|\xi_{l}\right| s_{n}^{-1} \leqslant \varepsilon\right.$, $1 \leqslant l \leqslant n\}, L_{n}(t)=\left\{\omega: s_{n}^{-1} \sum_{1}^{n} \xi_{l}=t\right\}$. Тогда, используя формулу полной вероятности и производя элементарные выкладки, последовательно получим

$$
\begin{aligned}
\mathbf{P}\left\{K_{n}(\varepsilon) \cap L_{n}(t)\right\}= & \int_{-\varepsilon s_{n}}^{\varepsilon s_{n}} \mathbf{P}\left\{K_{n-1}\left(\varepsilon c_{n}\right) \cap L_{n-1}\left(t_{n}(y)\right)\right\} \phi(y) d y \\
p_{n}(\varepsilon ; t)= & \frac{\mathbf{P}\left\{K_{n}(\varepsilon) \cap L_{n}(t)\right\}}{\phi(t) d t}=\int_{-\varepsilon s_{n}}^{\varepsilon s_{n}}\left(\frac{\phi\left(t_{n}(y)\right) d\left(t_{n}(y)\right)}{\phi(t) d t}\right) \\
& \times \frac{\mathbf{P}\left\{K_{n-1}\left(\varepsilon c_{n}\right) \cap L_{n-1}\left(t_{n}(y)\right)\right\}}{\phi\left(t_{n}(y)\right) d\left(t_{n}(y)\right)} \phi(y) d y \\
= & c_{n} \int_{-\varepsilon s_{n}}^{\varepsilon s_{n}} p_{n-1}\left(\varepsilon c_{n} ; t_{n}(y)\right) \phi\left(y_{n}(t)\right) d y \\
= & s_{n} \int_{a_{-}}^{a_{+}} p_{n-1}\left(\varepsilon c_{n} ; \frac{t}{c_{n}}-v\right) \phi\left(s_{n} v\right) d v,
\end{aligned}
$$

если ввести обозначения $t_{n}(y)=\left(s_{n} t-y\right) / s_{n-1}, y_{n}(t)=\left(s_{n} y-t\right) / s_{n-1}$.

Отметим, что третье равенство в цепочке является следствием того, что $\phi\left(t_{n}(y)\right) \phi(y) / \phi(t)=\phi\left(y_{n}(t)\right)$, поскольку $\left(t_{n}(y)\right)^{2}-t^{2}+y^{2}=\left(y_{n}(t)\right)^{2}$. А четвертое возникает после замены переменных $y_{n}(t)=s_{n} v$. Лемма доказана.

3 а м е ч ан и е 4. Аргумент $t / c_{n}-y$ подынтегральной функции $p_{n-1}$ в (33) лежит в интервале

$$
c_{n}(t-\varepsilon) \leqslant \frac{t}{c_{n}}-y \leqslant c_{n}(t+\varepsilon) ;
$$

скажем,

$$
\frac{t}{c_{n}}-a_{+}=\frac{(n-1) t-(n \varepsilon-t)}{s_{n} s_{n-1}}=\frac{n(t-\varepsilon)}{s_{n} s_{n-1}}=c_{n}(t-\varepsilon) .
$$

Теперь можно привести без доказательства искомое утверждение.

Следствие 6. Если у $\xi_{1}$ существует плотность, т.е. $d \mathbf{P}\left\{\xi_{1}<\right.$ $t\}=f(t) d t, u d \mathbf{P}\left\{\sum_{1}^{n} \xi_{l}<s_{n} t\right\}=f_{n}(t) d t$, mо при $n \geqslant 2$

$$
p_{n}(\varepsilon ; t)=c_{n} \int_{-\varepsilon s_{n}}^{\varepsilon s_{n}} p_{n-1}\left(\varepsilon c_{n} ; t_{n}(y)\right) \frac{f_{n-1}\left(t_{n}(y)\right) f(y)}{f_{n}(t)} d y, \quad|t| \leqslant n \varepsilon ;
$$

$p_{1}(\varepsilon ; t)=I(|t| \leqslant \varepsilon)$, а плотность $f_{n}(y)$ определяется рекуррентным соотношением

$$
f_{n}(t)=\int f_{n-1}\left(t_{n}(y)\right) f(y) d y, \quad n \geqslant 2 .
$$

6.3. Конкретный пример и некоторые уточнения. Рассмотрим далее ситуацию нормально распределенных с.в. леммы 8. Покажем, что в этом случае искомая условная вероятность, по определению равная нулю 
при $|t|>n \varepsilon$, может быть весьма просто записана с помощью функции распределения $\Phi(t)$ одномерной с.в. $N(0,1)$ на интервале $|t| \leqslant n \varepsilon$.

Лемма 9. В условиях леммы 8 при любом $\varepsilon>0$

$$
p_{n}(\varepsilon ; t)=\int_{A(n)} \prod_{1}^{n-1}\left[\phi\left(x_{l}\right) d x_{l}\right], \quad|t| \leqslant n \varepsilon
$$

$2 \partial e$

$$
\begin{aligned}
A(n) & =\left\{n \varepsilon_{-} \leqslant x_{1} \sqrt{n-1} \leqslant n \varepsilon_{+},\left|x_{l}\right| \leqslant 2 \varepsilon \sqrt{n(l-1) l^{-1}}, l=2, \ldots, n-1\right\}, \\
\varepsilon_{-} & =t n^{-1}-\min [\varepsilon,(n-1) \varepsilon+t], \quad \varepsilon_{+}=t n^{-1}+\min [\varepsilon,(n-1) \varepsilon-t] .
\end{aligned}
$$

Д ок а з а т ель с т о. Поскольку плотность с.в. $\xi_{l} / \sqrt{n}$ есть $\sqrt{n} \phi(t \sqrt{n})$, то

$$
p_{n}(\varepsilon ; t) \phi(t) d t=\left(\frac{n}{2 \pi}\right)^{n / 2} \int_{B(1)} \exp \left\{-\frac{n}{2} \sum_{1}^{n} y_{l}^{2}\right\} d y_{1} \cdots d y_{n}
$$

где $B(1)=\left\{\left|y_{l}\right| \leqslant \varepsilon, 1 \leqslant l \leqslant n ; \quad \sum_{1}^{n} y_{l}=t\right\}$. Далее сначала избавимся от элемента $\phi(t) d t$ в обеих частях приведенного равенства, используя представление

$$
\begin{aligned}
& \frac{n}{2}\left(\sum_{1}^{n-1} y_{l}^{2}+\left(t-\sum_{1}^{n-1} y_{l}\right)^{2}\right) \\
& \quad=\frac{t^{2}}{2}+n\left(\sum_{1}^{n-1}\left(\frac{t}{n}-y_{l}\right)^{2}+\sum_{i<j}^{n-1}\left(\frac{t}{n}-y_{i}\right)\left(\frac{t}{n}-y_{j}\right)\right),
\end{aligned}
$$

а затем проведем несколько замен переменных и получим:

$$
\begin{aligned}
& p_{n}(\varepsilon ; t)= \sqrt{2 \pi}\left(\frac{n}{2 \pi}\right)^{n / 2} \int_{B(2)} \exp \left\{-n\left[\sum_{1}^{n-1}\left(\frac{t}{n}-y_{l}\right)^{2}\right.\right. \\
&\left.\left.+\sum_{i<j}^{n-1}\left(\frac{t}{n}-y_{i}\right)\left(\frac{t}{n}-y_{j}\right)\right]\right\} d y_{1} \cdots d y_{n-1} \\
&=(2 \pi)^{-(n-1) / 2} \sqrt{n} \int_{B(3)} \exp \left\{-\left(\sum_{1}^{n-1} z_{l}^{2}+\sum_{i<j}^{n-1} z_{i} z_{j}\right)\right\} d z_{1} \cdots d z_{n-1} \\
&=(2 \pi)^{-(n-1) / 2} \sqrt{n} \int_{B(4)} \exp \left\{-\frac{1}{2}\left(n y_{1}^{2}+\sum_{2}^{n-1} y_{l}^{2}\right)\right\} d y_{1} \cdots d y_{n-1} \\
&=(2 \pi)^{-(n-1) / 2} \int_{A(n)} \exp \left\{-\frac{1}{2} \sum_{1}^{n-1} x_{l}^{2}\right\} d x_{1} \cdots d x_{n-1},
\end{aligned}
$$

где

$$
B(2)=\left\{\left|y_{l}\right| \leqslant \varepsilon, 1 \leqslant l \leqslant n-1, \varepsilon_{-} \leqslant \sum_{1}^{n-1}\left(y_{l}-\frac{t}{n}\right) \leqslant \varepsilon_{+}\right\},
$$




$$
\begin{aligned}
& B(3)=\left\{\left|z_{l}+\frac{t}{\sqrt{n}}\right| \leqslant \varepsilon \sqrt{n}, 1 \leqslant l \leqslant n-1, \varepsilon_{-} \sqrt{n} \leqslant \sum_{1}^{n-1} z_{l} \leqslant \varepsilon_{+} \sqrt{n}\right\}, \\
& B(4)=\left\{\varepsilon_{-} \sqrt{n} \leqslant y_{1} \sqrt{n-1} \leqslant \varepsilon_{+} \sqrt{n},\left|y_{l}\right| \leqslant 2 \varepsilon \sqrt{\frac{n(l-1)}{l}}, l \geqslant 2\right\} .
\end{aligned}
$$

В самом деле, первое равенство в (36) возникает после сокращения, а замена $\left(y_{l}-t / n\right) \sqrt{n}=z_{l}$ позволяет записать второе равенство. Отметим лишь, что запись множества $B(3)$ легко вытекает из приведенной записи множества $B(2)$, причем ее форма объясняется проведенной заменой, а иначе можно было бы представить ее в виде $B(2)=\left\{\left|y_{l}\right| \leqslant \varepsilon\right.$, $\left.1 \leqslant l \leqslant n-1,\left|t-\sum_{1}^{n-1} y_{l}\right| \leqslant \varepsilon\right\}$. Для перехода к третьему равенству используем ортогональное преобразование (см. $[5, \S 15$, отдел 3 , упражнение 1263]) $y_{1}=(1 / \sqrt{n-1}) \sum_{1}^{n-1} z_{l}, y_{l}=(1 / \sqrt{(l-1) l})\left[\sum_{1}^{l-1} z_{i}-(l-1) z_{l}\right]$, $l=2, \ldots, n-1$, при котором первая квадратичная форма в показателе экспоненты переходит во вторую. Что же касается множества $B(4)$, то для получения его записи достаточно использовать операции сложения неравенств и умножения их на число. Замену же, позволяюшую прийти к последнему равенству, объяснять не надо. Лемма доказана.

Следствие 7. В условиях леммь 9:

1) $p_{2}(\varepsilon ; t)=2 \Phi(2 \varepsilon-|t|)-1,|t| \leqslant 2 \varepsilon$ при любом $\varepsilon>0$;

2) функиия $p_{n}(\varepsilon ; t)$ является четной и выпуклой на отрезке $|t| \leqslant n \varepsilon$ $u$, значит, монотонно убывающей на интервале $0 \leqslant t \leqslant n \varepsilon$;

3) $p_{n}(\varepsilon ; t) \rightarrow 1, n \rightarrow \infty$ при фиксированном $t$, если $\varepsilon=((2 \ln n) / n)^{1 / 2}$.

7. Сравнение с представлением при условии Крамера. Укажем теперь вид ПБУ при условии Крамера из [2], с которым предлагается сравнивать полученный результат, а также приведем соображения, позволяющие утверждать, что эти представления сближаются не только при увеличении числа $n$ слагаемых нормированной суммы $S_{n} / s_{n}$, но и при «сближении» условия (2) с условием Крамера, т.е. при $s \rightarrow \infty$.

В [2] рассматривается та же последовательность н.о.р.с.в. $\left(X_{l}\right)$, удовлетворяюших условию (1). Однако там вместо условия (2) используется условие Крамера, причем в форме

$$
\varphi(\lambda) \equiv \mathbf{E} e^{\lambda X_{1}}<\infty, \quad 0 \leqslant \lambda<\Delta,
$$

где $\Delta>0$ - некоторое фиксированное число. Естественно, что, хотя взаимно однозначная связь параметров $x$ и $\lambda$ задается тем же равенством (5) и по-прежнему $\lambda \sigma=c \sigma(z)$, но определяюшие эти соотношения элементы определяются несколько иначе:

$$
m(z)=\frac{\varphi^{\prime}(z)}{\varphi(z)}, \quad \sigma^{2}(z)=\frac{\varphi^{\prime \prime}(z) \varphi(z)-\varphi^{\prime 2}(z)}{\varphi^{2}(z)}=d^{2} \sigma^{2} .
$$


Теорема 1 (см. [2]). Если выполнены условия (1), (2а), то при всех $0<\lambda<s_{n} \Delta=d \Delta \sqrt{n}$ и соответствуюших им значениях $x$ из (5)

$$
\mathbf{P}\left\{S_{n}>x s_{n}\right\}=\frac{\varphi^{n}(z) e^{-\lambda x}}{c \sigma(z) \sqrt{2 \pi}}\left(1+\delta_{n}(\lambda)\right),
$$

$\partial \partial$

$$
\delta_{n}(\lambda)=2 \theta \nu c \sigma(z) \sqrt{2 \pi}-[1-J(c \sigma(z))] .
$$

Итак, сами ПБУ (11), допустимые интервалы для $\lambda$, выражения для $x$ и ошибки $\delta_{n}(\lambda)$ очень похожи. В то же время видны и отличия, появившиеся после замены условия (2a) на (2). ІІрокомментируем их кратко, учитывая, что хотя теорема и сформулирована для фиксированных $n$, но ее форма скорее предназначена для ситуаций, когда $n \rightarrow \infty$.

Как и в (12a), в выражении (12) по-прежнему присутствуют две составляющих ошибки: первое слагаемое характеризует «близость» с.в. $H$ к предельному нормальному закону, т.е. должно стремиться к 0 при $n \rightarrow \infty$, а второе слагаемое отражает зависимость ошибки от параметра $\lambda$ и должно стремиться к 0 при $\lambda \rightarrow \infty$. Как показано в [2], именно так обстоят дела в случае условия Крамера. Можно ли сказать то же сейчас?

Прежде всего, ясно, что элементы $\Delta_{n}$ могут быть сделаны сколь угодно малыми увеличением $\lambda, T$ и $n$ (поскольку $\mathbf{P}(\bar{A}) \rightarrow 0, n \rightarrow \infty$ ). Понятно и то, что коэффициент $\delta_{1} e^{\mu_{1}}$ при $J(\cdot)$ стремится к 1 при том же выборе тех же параметров. Однако не все так гладко.

Дело в том, что $c \sigma(z) \sim \lambda$, поскольку $z \rightarrow 0$, а $\lambda$ может возрастать вместе с $n$. Поэтому желательно также иметь $\lambda \Delta_{n} \rightarrow 0$ при $\lambda \rightarrow \infty$ (остаюшееся слагаемое $c \sigma(z) \nu=O(z) \rightarrow 0)$. Но оказалось, что этого несложно добиться некоторым увеличением $\alpha$. Точнее говоря, для первого слагаемого в $\Delta_{n}$ это имеет место, поскольку $\lambda e^{-\lambda \sigma T} \rightarrow 0$ при $\lambda \rightarrow \infty$. Второе же слагаемое будет удовлетворять нашему требованию при $\alpha>3 /(2 s+3)$. В самом деле, в доказательстве леммы 3 соотношение $1-p=o(1)$ вытекало из неравенства $(2 s+2) \beta-2 s<0$. Теперь же требуется иметь $\lambda(1-p)=o(1)$. Поэтому достаточно положить $(2 s+2) \beta-2 s+1-\alpha<0$ и вспомнить, что $1-\alpha \leqslant \beta$.

Проблема сближения ПБУ при $s \rightarrow \infty$, по-видимому, требует специального исследования. Однако и сейчас можно отметить два соображения. Первое касается только что рассмотренной проблемы сближения ошибок, в отношении которой ясно, что при $s \rightarrow \infty$ сохранению свойства $\delta_{n}(\lambda)=o(1)$ может помешать лишь второе слагаемое в $\Delta_{n}$. Да и то лишь потому, что оценка леммы 5 слишком груба (представляется, что в тех же условиях $\mathbf{E}(\rho / \bar{A}) \sim 1, n \rightarrow \infty$, для любого $s)$. Тем не менее и с оценкой $\mathrm{E}(\rho / \bar{A})=O(\sqrt{s})$ можно получить желаемое. Скажем, если положить $\sqrt{s}=O\left((\sqrt{n})^{1-\alpha}\right)$, то проблему решает выбор $\alpha>2 /(s+2)$. 
Еще один момент касается перехода области задания $\lambda<C(\sqrt{n})^{1-\alpha}$ в предельную $\lambda<d \Delta \sqrt{n}$. Так вот, с помощью теоремы 2 из [2] легко выявляется причина, по которой на месте $C$ естественно ожидать появления величины $d \Delta$. Остальное же понятно: в силу (10) можно считать, что $\alpha \rightarrow 0, s \rightarrow \infty$.

Заключение. Конечно, громоздкое доказательство является недостатком, поскольку в нем «легче» где-то допустить ошибку и не заметить ее. Более того, интересный вопрос задал анонимный рецензент, которым он вообще ставил под сомнение справедливость утверждения теоремы. Правда, реальность противоречия между формулой (11) и указанным в замечании рецензента следствием из давней работы И.Ф. Пинелиса несколько сама была поставлена под сомнение тем, что автору не удалось найти его доказательства. Поэтому выскажем ряд соображений, все-таки позволивших автору принять твердое решение опубликовать эту работу.

Прежде всего, представляется, что рассматриваемая здесь проблема весьма важна для всей проблематики БУ и потому уже давно стоило ее поднять в открытой печати. Поэтому даже если допустить, что предлагаемый результат ошибочен, то «сообща легче найти ошибку» и тем самым, возможно, либо уточнить условия существования искомого представления, либо понять причину того, что оно не существует, т.е. решить серьезную проблему. А это, в принципе, наша общая цель.

Во-вторых, автор постарался заметно упростить первоначальный вариант доказательства и тщательно его проверить. Наконец, в третьих, подчеркнем на наш взгляд основной элемент, который отличает предложенное ПБУ от «крамеровского» и который позволяет верить, что данный результат верен. Дело в том, что сами выражения ПБУ (11) и (11a) действительно «асимптотически примерно одинаковы», но не следует забывать, что эта «одинаковость» относится к интервалу $0<\lambda<C(\sqrt{n})^{1-\alpha}$, составляющему при $\alpha>0$ в пределе «нулевую» долю от интервала $0<\lambda<d \Delta \sqrt{n}$, для которого установлено ПБУ (11a).

\section{СПИСОК ЛИТЕРАТУРЫ}

1. Жуленев С. В. О больших уклонениях, І. - Теория вероятн. и ее примен., 1999, т. 44 , в. 1 , с. $34-54$.

2. Жуленев С. В. Большие уклонения. Простейшая ситуация. - Вестн. Моск. ун-та, 2005, № 1, c. 16-25.

3. Саулис Л., Статулявичус В. Предельные теоремы о больших уклонениях. Вильнюс: Мокслас, 1989, 206 с.

4. Ширяев А. Н. Вероятность. М.: Наука, 1980, 578 с.

5. Проскуряков И. В. Сборник задач по линейной алгебре. М.: Наука, 1970, 384 с. 